\title{
Unterschiedliche Taxpunktwerte in Arztpraxen und Spitälern: Warum?
}

\author{
Jacques-Henri Weidmann, Tarifdienst FMH
}

Obwohl die TARMED-Tarifstruktur einheitlich ist, fragen sich viele, warum der Taxpunktwert in der privaten ambulanten Arztpraxis anders ist als im ambulanten Bereich der Spitäler. Die Erklärung ist in zwei Bereichen zu suchen.

\section{KVG}

Die Taxpunktwerte werden kantonal oder regional festgelegt. Grundsätzlich gibt es mindestens zwei Tarifgemeinschaften oder Budgets pro Kanton. Die eine bindet die Ärzte über die kantonale Ärztegesellschaft an santésuisse, die andere H+ an santésuisse ebenfalls auf kantonaler oder regionaler Basis. In diesem Fall wird auch zwischen öffentlichen und privaten Spitälern unterschieden.

Die Festlegung des Taxpunktwerts bei der Einführung des TARMED erfolgte gestützt auf die Transcodierung des kantonalen Tarifs in den TARMED. Dieser Vorgang wurde auch für den ambulanten Spitaltarif durchgeführt. So wurde eine Gesamtzahl der in einem Jahr in Rechnung gestellten Punkte bestimmt. Da das Kostenneutralitätskonzept ein jährliches Fakturierungsvolumen vorgibt, wurde dieses durch die in einem Jahr verrechnete Gesamtzahl an Punkten geteilt. Das Ergebnis ist ein Taxpunktwert, der sich zwangsläufig zwischen der Privatpraxis und der ambulanten Spitalpraxis unterscheidet.

Die einzige Ausnahme betrifft den Kanton Genf, wo eine Tarifgemeinschaft die Ärzte, H+ und santésuisse umfasst.

Dazu kommt die Tatsache, dass die Ärzte und santésuisse über Daten verfügen, die ein echtes Management der Kostenneutralität durch das Kostenneutralitätsbüro ermöglichen. Dies führt zu regelmässigen Anpassungen des Taxpunktwerts für die freipraktizierenden Ärzte. Umgekehrt kann jedoch mit den verfügbaren Daten der Taxpunktwert im Budget $\mathrm{H}+/$ santésuisse noch nicht verwaltet werden.

Neben den im Gesetz verankerten Begriffen Wirksamkeit, Zweckmässigkeit und Wirtschaftlichkeit lösen diese Unterschiede beim Taxpunktwert innerhalb eines Kantons bei den
Bürgern die Frage aus, weshalb die gleiche Leistung je nach dem Status des Leistungserbringers zu sehr unterschiedlichen Fakturierungen führen kann. Ebenso erstaunlich ist, dass die öffentlichen, vom Staat subventionierten Spitäler im allgemeinen einen höheren Taxpunktwert geniessen als die freipraktizierenden Ärzte, die keine staatlichen Beihilfen erhalten.

\section{UVG/IVG/MVG}

Hier sind die Leistungserbringer und Versicherer durch zwei Verträge aneinander gebunden. Der eine besteht mit den Ärzten (FMH), der andere mit den Spitälern (H+). Zum Zeitpunkt der Einführung des Tarifs, d.h. am 1. Mai 2003 für die freipraktizierenden Ärzte und am 1. Januar 2004 für die Spitäler, wurde der Taxpunktwert in beiden Fällen auf Fr. 1.- festgelegt. Die fallkostenstabile Periode von 18 Monaten begann am Tag der Tarifeinführung, und am Ende wurde der Taxpunktwert für die freipraktizierenden Ärzte auf Fr. -.92 gesenkt. Bei den Spitälern dauert diese Periode noch bis am 30. Juni 2005.

Deswegen gibt es zurzeit zwei unterschiedliche Taxpunktwerte in Spitälern und privaten Arztpraxen. Leider auch in diesem Fall muss der Arzt mit dem niedrigeren Taxpunktwert fakturieren. Diese Situation ist aber möglicherweise nur von vorübergehender Dauer bis am 30. Juni 2005.

\section{Fazit}

Die TARMED-Rahmenverträge (KVG und UVG/ IVG/MVG) legen getrennte Tarifgemeinschaften für Ärzte und Spitäler fest. Die daraus folgenden Taxpunktwerte unterscheiden sich für jeden Leistungserbringer. Deshalb sollte eine Konvergenz des Taxpunktwerts nicht nur zwischen den Kantonen, sondern auch zwischen den verschiedenen Leistungserbringern erfolgen, um die Tarife zu harmonisieren und so sinnlose Anreize zu vermeiden. 